\title{
Lentivirus-mediated expression of Drosophila melanogaster deoxyribonucleoside kinase driven by the hTERT promoter combined with gemcitabine: A potential strategy for cancer therapy
}

\author{
NIANQU ZHANG ${ }^{1}$, LEI ZHAO ${ }^{2}$, SHUAI MA ${ }^{1}$, MING GU $^{1}$ and XINYU ZHENG ${ }^{1,3}$ \\ ${ }^{1}$ Department of Surgical Oncology and Department of General Surgery, The First Affiliated Hospital, ${ }^{2}$ Center of Experimental \\ Technology and Medical Research, ${ }^{3}$ Laboratory 1 , Cancer Institute, China Medical University, Shenyang, P.R. China
}

Received March 15, 2012; Accepted May 28, 2012

DOI: $10.3892 /$ ijmm.2012.1033

\begin{abstract}
In contrast to other enzymes, Drosophila melanogaster deoxyribonucleoside kinase $(D m-d N K)$ has a broad substrate specificity and high catalytic rate when transferred in human cells. This makes it a promising therapeutic agent when administered together with several cytotoxic nucleoside analogs, such as gemcitabine 2',2'-difluoro-deoxycytidine $(\mathrm{dFdC})$. Therefore, lentiviral vectors, which potentially allow stable long-term transgene expression, are good candidates for gene delivery vehicles. In the present study, we successfully developed a lentivirus-mediated transgene expression system of $D m-d N K$ under the control of hTERT promoter against the breast cancer cell line (Bcap37), the gastric cancer cell line (SGC7901) and the normal fibroblast cell line (WI-38). Moreover, we also analyzed its targeted cytotoxicity in vitro with treatment of the prodrug $\mathrm{dFdC}$. Bcap37 tumor growth was inhibited in nude mice. Both cancer cell lines exhibited apparent cytotoxicity when infected with recombinant lentivirus constructs expressing $D m-d N K$. In contrast, lentivirus-infected WI-38 cells exhibited less cytotoxicity. These data suggested that $D m-d N K$ was sensitive to $d F d C$, and it resulted in synergistic growth inhibition and apoptosis induction in vitro. In addition, Lenti-hTERT-dNK/dFdC also suppressed tumor growth in vivo. Our results suggest that the Lenti-hTERT-dNK/dFdC system is a safe and feasible treatment strategy in the development of suicide gene therapy.
\end{abstract}

\section{Introduction}

Over the past decade, suicide gene therapies have been widely studied in a broad array of human cancer types. This strategy

Correspondence to: Dr Xinyu Zheng, Department of Surgical Oncology and Department of General Surgery, The First Affiliated Hospital, China Medical University, 155 North Nanjing Street, Shenyang 110001, P.R. China

E-mail: xyzheng@mail.cmu.edu.cn

Key words: Drosophila melanogaster deoxyribonucleoside kinase, lentivirus, hTERT promoter, nucleoside analog, suicide gene involves the transfer of suicide genes into cancer cells, and followed by harmless prodrug treatment. A common approach used is the herpes simplex virus thymidine kinase/ganciclovir (HSV-TK/GCV) system, and its clinical trials are in the phase III stage. According to these findings, multisubstrate deoxyribonucleoside kinase of Drosophila melanogaster $(D m-d N K)$ has received noticeable attention as another potential therapeutic agent for cancer treatment. Similar to herpes simplex virus deoxyribonucleoside kinase, $D m-d N K$ first catalyzes the nucleoside analog into its monophosphate form, which is considered the rate-limiting step throughout the activation. This monophosphate form is further catalyzed into other phosphorylated forms by normal cellular kinases, resulting in the arrest of DNA replication and induced cell apoptosis. $D m-d N K$ as a suicide gene also displays increased sensitivity to several nucleoside analogs. Bertoli et al (1) reported that pyrimidine nucleoside analogs (E)-5-(2-bromovinyl)-2'deoxyuridine (BVDU) and 1-b-D-arabinofuranosylthymine (araT) are valuable for enhancing the efficacy of $D m-d N K$ suicide gene therapy. Knecht et al (2)demonstrated that gemcitabine 2',2'-difluoro-deoxycytidine (dFdC), an anticancer drug, is an efficient substrate for $D m-d N K$, which can efficiently phosphorylate $\mathrm{dFdC}$, and the $2.2 \AA$ resolution structure of $D m-d N K$ is important. These data reveal that the residues Tyr70 and Arg105 take into consideration the positioning of $\mathrm{dFdC}$, suggesting its significant implications for therapeutic efforts due to the broad substrate acceptance of $D m-d N K$. Characterized by its high catalytic property and broad substrate acceptance, $D m-d N K$ has therapeutic potential and is used in the treatment of several cancer types (3-5).

Despite these advantages, one issue is the limited efficiency, suggesting that the discovery of new approaches is urgently required. A previous study has demonstrated an approach to selectively kill c-Myc-expressing lung cancer cells by fusing the c-Myc gene promoter with TK gene (6). In particular, in order to increase the potency of efficient killing in a wide range of human cancers, human telomerase reverse transcriptase (hTERT) serves as an ideal biomarker, and hTERT promoterdirected suicide gene therapy can be employed as a modality for a targeted suicide gene therapy for hTERT-positive tumors $(7,8)$. In the present study, we investigated the selective tumor 
cell killing of hTERT promoter in the treatment of human cancers. We selected the hTERT promoter to drive $D m-d N K$ gene expression and thus generated a tumor-selective viral vector through $\mathrm{dFdC}$ administration. Recently, adenoviral vector-mediated $D m-d N K$ expression has been established and is considered auspicious (9). However, its clinical use is still limited due to the short-term expression and vector-induced toxicity. With respect to the application of efficient and safe gene delivery vehicles, lentiviral vectors have become potentially useful vehicles since they provide stable and long-term expression of the transgene by incorporation into the target cell DNA. Therefore, the feasibility of hTERT promoter-induced $D m-d N K$ gene expression via lentiviral vector is expected. In our present study, we first developed a Lenti-hTERT-dNK/ $\mathrm{dFdC}$ system and tested its effect on the targeted therapeutic approach both in vitro and in vivo. Our results demonstrated that this system could be a valuable tumor targeting strategy for tumor control.

\section{Materials and methods}

Cells and culture. Human breast cancer cell line Bcap37 and human gastric cancer cell line SGC7901 were both obtained from the Cancer Institution of China Medical University (Shenyang, China). Normal human fetal lung fibroblast cell line WI-38 was purchased from ATCC (American Type Cell Culture, Manassas, VA, USA). Bcap37 was cultured in Roswell Park Memorial Institute (RPMI)-1640 medium. SGC7901 and WI-38 were cultured in high glucose Dulbecco's modified Eagle's medium (DMEM). Cells were maintained in a medium supplemented with $10 \%$ heat inactivated fetal bovine serum (FBS) (Gibco-BRL, Germany), 1\% L-glutamine, $100 \mathrm{U} / \mathrm{ml}$ penicillin and $100 \mathrm{U} / \mathrm{ml}$ streptomycin at $37^{\circ} \mathrm{C}$ in a humidified incubator supplied with $5 \% \mathrm{CO}_{2}$.

Construction of the lentiviral vectors. The Dm-dNK coding sequence was amplified from plasmid PLXSN-dNK using a polymerase chain reaction (PCR) technique with the following primers: upstream, 5'-CCG GAA TTC (EcoRI) ACC ATG GCG CAG GCA-3' and downstream, 5'-CGC GGA TCC (BamH) TCA TTA TCT GGC GAC-3'. The synthetic DNA sequence was released with endonucleases EcoRI and BamH (New England Biolabs, Beverly, MA, USA). Dm- $d N K-3$ Flag was amplified by PCR and ligated into plasmid PGC-FU (GeneChem, Shanghai, China) consisting of a 5'-long terminal repeat (LTR), cytomegalovirus (CMV) promoter, multiple clone site, a green fluorescent protein (GFP) sequence and a 3'-LTR. Moreover, $D m-d N K$-3Flag was also ligated into plasmid PGC-FU-hTERT (GeneChem) consisting of a 5'-LTR, hTERT promoter, multiple clone site, GFP sequence and a 3'-LTR. In order to generate the recombinant plasmid PGC-FU-dNK or PGC-FU-hTERT-dNK, GFP was removed with endonucleases $A g e \mathrm{I}$ and $E c o$ RI (New England Biolabs Ltd., UK). Subsequently, the plasmids together with two packaging plasmids PHelper1.0 (gag, pol and rev, component) and plasmid PHelper2.0 (VSVG, component) were packed and co-transfected into a human embryonic kidney cell line (HEK293T) using Lipofectamine ${ }^{\mathrm{TM}} 2000$ reagent (Invitrogen, USA) according to the manufacturer's instructions. Lentivirus-containing medium was filtered from the post-transfection supernatant and used for transductions. Concentrated viruses of Lenti-GFP, Lenti-hTERT-dNK and Lenti-CMV-dNK were obtained, respectively, and then stored at $-80^{\circ} \mathrm{C}$. The titer of lentiviral vectors was determined by dilution. Lenti-GFP was used to determine the infectivity between cell lines. In order to increase the infection efficiency, all lentivirus-infected cells were cultured in the medium containing Polybrene $(6 \mu \mathrm{g} / \mathrm{ml})$ (Sigma, USA).

RT-PCR. Bcap37, SGC-7901 and WI-38 cells were seeded in 6-well plates at a density of $10^{5}$ cells/well for $24 \mathrm{~h}$, respectively. Then $D m-d N K$ at a multiplicity of infection (MOI) of 10 was added to the cell cultures. Polybrene $(6 \mu \mathrm{g} / \mathrm{ml})$ was added to all cultures. Total-RNA was extracted from the cultured cells using TRIzol reagent (Sigma) 3 days after infection. In order to minimize the genomic DNA contamination, purified RNA was treated with DNase I. cDNA was synthesized from 1 ng of RNA using the RT-PCR kit (Takara Bio, Inc., Japan) following the manufacturer's protocol. Human glyceraldehyde-3-phosphate dehydyrogenase (GAPDH) was used as the housekeeping gene. Amplification was performed using PCR with the following primers: $D m-d N K$, upstream, 5'-CCG GAA TTC ACC ATG GAG GCA-3' and downstream, 5'-CGC GGA TCC TCA TTA TCT GGC GAC-3'; and GAPDH, upstream, 5'-ACC ACA GTC CAT GCC ATC AC-3' and downstream, 5'-TCC ACC ACC CTG TTG CTG TTG CTG TA-3'. Briefly, following a pre-heating step at $94^{\circ} \mathrm{C}$ for $4 \mathrm{~min}, D m-d N K$ was amplified with 35 cycles at a melting temperature of $94^{\circ} \mathrm{C}$ for $1 \mathrm{~min}$, an annealing temperature of $60^{\circ} \mathrm{C}\left(55^{\circ} \mathrm{C}\right.$ for GAPDH $)$ for $1 \mathrm{~min}$, and an extension temperature of $72^{\circ} \mathrm{C}$ for $1.5 \mathrm{~min}$. The expected length of $D m-d N K$ amplicon was $779 \mathrm{bp}$. Relative expression of $D m-d N K$ was normalized to GAPDH (452 bp). A total of $10 \mu 1$ of each amplicon was separated by electrophoresis on a $2 \%$ agarose gel, and the efficiency of cDNA synthesis was determined by PCR with GAPDHspecific primers and visualized by SYBR-Green staining.

Western blotting. Cells were harvested from 6-well plates after transfection as previously described. For protein detection, the cells were lyzed with lysis buffer $(50 \mathrm{mmol} / \mathrm{l}$ HEPES at $\mathrm{pH} 7.4,250 \mathrm{mmol} / \mathrm{l} \mathrm{NaCl}, 1 \mathrm{mmol} / \mathrm{l} \mathrm{NaF}, 1 \mathrm{mmol} / \mathrm{l} \mathrm{EDTA}$, $1 \%$ Triton X-100, $1 \mathrm{mmol} / \mathrm{l}$ DTT) containing protease inhibitors. The cell lysates were then normalized for protein content using the BCA protein assay kit (KeyGEN, Shanghai, China). Equal amount of protein containing sample buffer was separated on SDS-polyacrylamide gel by electrophoresis and then transferred to polyvinylidene difluoride (PVDF) membranes (Millipore, Bedford, MA, USA). The membranes were blocked in Tris-buffered saline (TBS) $(10 \mathrm{mM}$ Tris- $\mathrm{HCl}$ at $\mathrm{pH} 8.0,0.05 \%$ Tween-20, $150 \mathrm{mM} \mathrm{NaCl}$ ) containing $1 \%$ bovine serum albumin (BSA) at room temperature for $2 \mathrm{~h}$ and then probed with antiFlag $(1: 1,000)$ (Abcam, Cambridge, CA, USA) or anti- $\beta$-actin (1:500), which was followed by a secondary horseradish peroxidase-conjugated antibody (Santa Cruz Biotechnology, Inc., Santa Cruz, CA, USA). After several washes, the corresponding blots were developed with a chemiluminescence reagent (Thermo Fisher Scientific, Inc., Rockford, IL, USA). $\beta$-actin was used as a control for equal protein loading.

Enzyme assays. Cell protein extracts were prepared as described (10) from Bcap37, SGC7901 and WI-38 cells at $72 \mathrm{~h}$ after 
lentivirus infection. The activity of purified recombinant enzymes was determined in a 35-ml reaction mixture containing $50 \mathrm{mM}$ Tris- $\mathrm{HCl}$ at $\mathrm{pH} 7.6,5 \mathrm{mM} \mathrm{MgCL}_{2}, 2 \mathrm{mM}$ dithiothreitol, $15 \mathrm{mM} \mathrm{NaF}, 100 \mathrm{mM}$ KCL, $5 \mathrm{mM}$ ATP, $0.5 \mathrm{mg} / \mathrm{ml} \mathrm{BSA}$ and $0.6 \mathrm{mg}$ protein extract. Briefly, $2.5 \mathrm{mM}$ [methyl- $\left.{ }^{3} \mathrm{H}\right] \mathrm{dThd}$ (Moravek Biochemicals, Inc., Brea, CA, USA) was mixed with an equivalent amount of unlabeled substrate. Sample aliquots were spotted on Whatman DE-81 filter paper disks after incubation of 10,20 and $30 \mathrm{~min}$ at $37^{\circ} \mathrm{C}$, respectively. The filters were dried for $1 \mathrm{~h}$ and then washed three times in $5 \mathrm{mM}$ ammonium formate. The filter-bound nucleoside monophosphates were eluted with $0.5 \mathrm{M} \mathrm{KCl}$, and the radioactivity was measured by scintillation counting.

Cell viability analysis. Exponentially growing cells were seeded into 96-well plates (Corning Inc., USA). After overnight culture, cells were infected with Lenti-GFP, LentihTERT-dNK, Lenti-CMV-dNK and untreated at the MOI rate of 10 . After 3 days, the infecting medium was replaced with fresh medium containing $10 \%$ FBS and $\mathrm{dFdC}$ at various concentrations from 0.001 to $10 \mu \mathrm{M}$. Then the plates were incubated at $37^{\circ} \mathrm{C}$ for $72 \mathrm{~h}$ in the humidified incubator supplied with $5 \% \mathrm{CO}_{2}$. Subsequently, $20 \mu \mathrm{l}$ of tetrazolium salt 3-(4,5-dimethylthiazol-2-yl)-2,5-diphenyl tetrazolium bromide (MTT) (Promega, USA) $(5 \mathrm{mg} / \mathrm{ml})$ was added to each well, and the plate was then incubated for $4 \mathrm{~h}$. The cells were lysed in $200 \mu \mathrm{l}$ of dimethylsulphoxide (DMSO) and mixed thoroughly. Color reaction was performed by determining the absorbance at $570 \mathrm{~nm}$, and the number of viable cells was measured. Each experiment was performed in triplicate and repeated three times.

Cell proliferation and flow cytometric apoptosis assays. All cells transfected with Lenti-GFP, Lenti-hTERT-dNK and Lenti-CMV-dNK or untreated cells were seeded in 24-well plates with a pre-coat of $\mathrm{dFdC}(1 \mu \mathrm{M})$. During the incubation, cells were trypsinized and suspended in a serum-free medium. Cell counting was carried out under a microscope using a hemocytometer. The cell numbers were obtained from an average of three experiments. To further evaluate the apoptosis extent and clarify the mechanism of this suicide gene therapy, an Annexin V-FITC/propidium iodide (PI) double staining kit (GenMed BioScience, China) was used to detect the apoptosis ratio. Briefly, Bcap37, SGC7901 and WI-38 cells were cultured in 6-well plates and incubated with $1 \mu \mathrm{M} \mathrm{dFdC} \mathrm{for} 72 \mathrm{~h}$ after the infection with Lenti-hTERT-dNK, Lenti-CMV-dNK, Lenti-GFP and untreated respectively for $72 \mathrm{~h}$. The cells were then harvested by trypsinization, washed twice with cold PBS and centrifuged at room temperature for $10 \mathrm{~min}$. After the supernatant was discarded, the cell pellet was resuspended with $200 \mu \mathrm{l}$ of $1 \mathrm{X}$ binding buffer with addition of $5 \mu \mathrm{l}$ Annexin V-FITC $(20 \mu \mathrm{g} / \mathrm{ml})$. Following a 15-min incubation in the dark, $200 \mu \mathrm{l}$ binding buffer and $10 \mu \mathrm{l}$ of PI $(50 \mu \mathrm{g} / \mathrm{ml})$ were added to the cell suspension. The percentage of cells undergoing apoptosis was then determined by a FACScan flow cytometer [equipped with CellQuest and ModFITLT for Mac V1.01 software (Becton-Dickinson, San Jose, CA, USA)].

Antitumor effect of Lenti-hTERT-dNK/dFdC system in vivo. To detect the antitumor effectiveness of Lenti-hTERT-dNK/
$\mathrm{dFdC}$ system in vivo, 24 female $\mathrm{BALB} / \mathrm{C}$ nude mice, 6- to 7-weeks old were purchased from the Experimental Animal Center, Chinese Academy of Sciences (Shanghai, China). All experiments followed the Guide for the Care and Use of Laboratory Animals (National Research Council, 1996). A total of $1.0 \times 10^{7}$ Bcap37 cells were suspended in $100 \mu 1 \mathrm{PBS}$ and then inoculated in the flanks of nude mice. Once the tumor reached a volume of $\sim 100 \mathrm{~mm}^{3}$, mice were randomly assigned into 4 groups (6 per group) as follows: i) $\mathrm{dFdC}$ with PBS; ii) $\mathrm{dFdC}$ with Lenti-GFP; iii) $\mathrm{dFdC}$ with Lenti-hTERT-dNK and iv) $\mathrm{dFdC}$ with Lenti-CMV-dNK. The animals in the lentivirus treatment groups received a series of intratumoral injections at a dose of $10^{9}$ plaque forming units (pfu) three times with a 2-day interval. Subsequently, $5 \mathrm{mg} / \mathrm{kg} \mathrm{dFdC} \mathrm{was} \mathrm{daily} \mathrm{admin-}$ istered into the peritoneal cavity over 7 consecutive days. Xenograft tumor burdens were inspected every 5 days and recorded with a caliper for up to 30 days. Tumor volume was calculated according to the formula: $\left(1 / 2 \mathrm{x}\right.$ length $\mathrm{x}$ width $\left.{ }^{2}\right)$.

Statistical analysis. All data are expressed as mean \pm SD and analyzed using the statistical software SPSS version 10.1 (SPSS, Inc., Chicago, IL, USA). P $<0.05$ was considered statistically significant.

\section{Results}

Dm-dNK expression. In order to determine the infection efficiency, we used lentivector plasmids PGC-FU, PGC-FUdNK-3Flag and PGC-FU-hTERT-dNK-3Flag. To construct plasmid PGC-FU-dNK-3Flag, the GFP expression cassette in plasmid PGC-FU was replaced by a dNK-3Flag expression cassette. We also constructed a plasmid PGC-FU-hTERTdNK-3Flag, in which the $D m-d N K$ gene was under the control of the hTERT promoter after the CMV promoter was removed from the plasmid PGC-FU-dNK-3Flag (Fig. 1A). The infectivity of the cell lines was determined by GFP expression in the Lenti-GFP. Fig. 1B shows that $>90 \%$ of the cancer cells (Bcap37 and SGC7901) and normal cells (WI-38) were transduced with Lenti-GFP at an MOI of 10. Then we analyzed the expression of $D m-d N K$ at the mRNA and protein levels by RT-PCR and western blotting, respectively. The null lentivirus and untreated control induced cells did not exhibit $D m-d N K$ expression at both the mRNA and protein levels. The $D m-d N K$ expression at the mRNA level was not significantly different in the cancer cells (Bcap37 and SGC7901) infected with LentihTERT-dNK and Lenti-CMV-dNK (Fig. 2A). The Dm-dNK expression at the protein level was slightly decreased in the Bcap37 cells infected with Lenti-hTERT-dNK compared with that of Lenti-CMV-dNK-infected cells, whereas it was nearly identical at the protein level in the SGC7901 cells infected with Lenti-hTERT-dNK and Lenti-CMV-dNK (Fig. 2B). In contrast, we also observed that the $D m-d N K$ expression at the mRNA and protein levels was low or completely absent in WI-38 cells.

To further evaluate the essential role of nucleoside kinase in the infected cells, we determined the phosphorylation of dThd in the cell protein extracts. As expected, the enzymatic activity of nucleoside kinase was strong in the Bcap37 and SGC7901 cells infected with Lenti-hTERT-dNK, which was $\sim 2$-fold higher than that in the cells infected with the lentiviral 
A

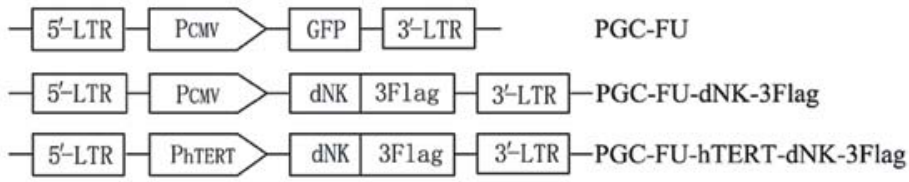

B

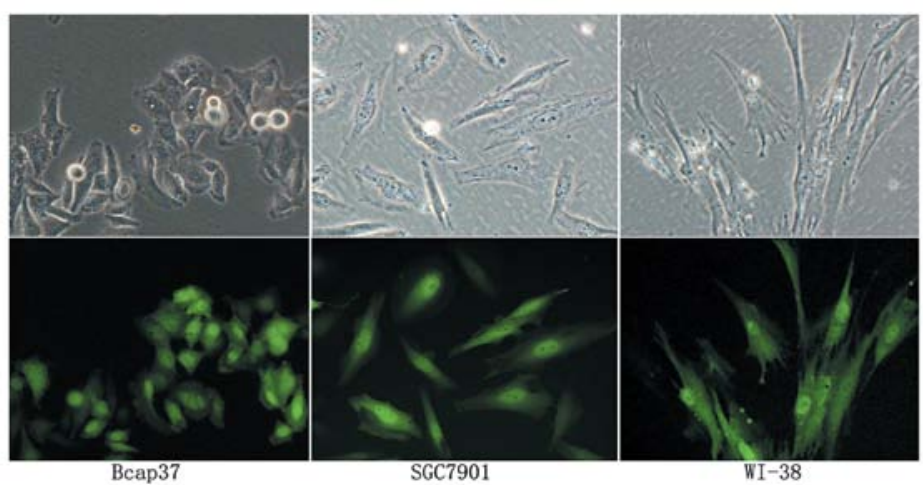

Figure 1. Lentiviral expression construct and GFP expression. (A) Schema of the three lentiviral constructs. GFP expression cassette in plasmid PGC-FU was replaced with cDNA encoding dNK-3Flag to construct PGC-FU-dNK-3Flag. CMV promoter in plasmid PGC-FU-dNK-3Flag was replaced with hTERT promoter to construct PGC-FU-hTERT-dNK-3Flag. (B) Fluorescence of GFP expressed from lentiviral vectors was observed as a marker for infection efficiency in cancer cell lines (Bcap37 and SGC7901) and normal cell line (WI-38). More than 90\% of the cells were transduced with Lenti-GFP at an MOI of 10.
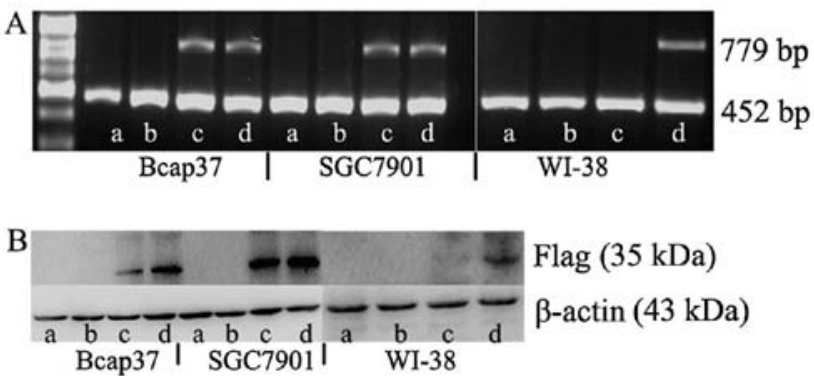

Figure 2. RT-PCR and western blot analysis of $D m-d N K$ expression at the mRNA and protein levels. (A) The analysis was performed in cells infected with (a) control, (b) Lenti-GFP, (c) Lenti-hTERT-dNK, (d) Lenti-CMV-dNK at an MOI of 10 for $72 \mathrm{~h}$ after infection. The expression of $D m-d N K$ at the mRNA level was determined by RT-PCR in Bcap37, SGC7901 and WI-38 cell lines. The 779- and 452-bp markers indicate the sizes of amplified Dm-dNK and GAPDH, respectively. (B) Western blot analysis was used to analyze the flag-tagged recombinant $D m-d N K$ at the protein level in cancer cell lines (Bcap37 and SGC7901) and normal cell line (WI-38), and $\beta$-actin was used as a negative control.

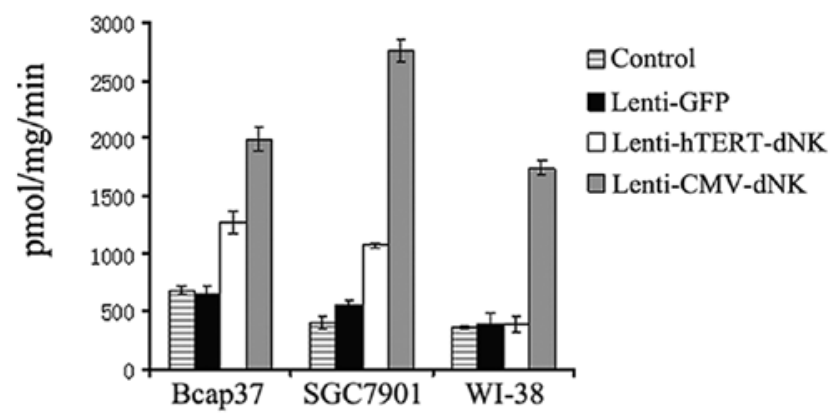

Figure 3. Dm- $d N K$ enzyme activity in cell protein extracts. The level of $D m-d N K$ activity was determined by quantifying the dThd phosphorylation in the cells. Dm- $d N K$ activity in the cancer cells (Bcap37 and SGC7901) infected with Lenti-hTERT-dNK was $\sim 2$-fold higher than that in the mock cells. In contrast, the $D m-d N K$ activity in WI-38 cells infected with LentihTERT-dNK was not different from that of its mock cells. vector alone. Although the $D m-d N K$ activity of the hTERT promoter in the Bcap37 and SGC7901 cells was consistently lower than that of the CMV promoter, the incidence of activity with the hTERT promoter was similar to that with the CMV promoter. In contrast, the $D m-d N K$ activity in WI-38 cells infected with Lenti-hTERT-dNK was not different from that of its mock cells (Fig. 3). These results were consistent with its expression levels in the previous study, establishing a connection between the enzymatic activity of $D m-d N K$ and its expression at the mRNA and protein levels in these experimental cell lines.

Cell cytotoxicity, proliferation and induction of apoptosis. In order to evaluate the effects of Lenti-hTERT-dNK on the prodrug $\mathrm{dFdC}$ in the infected cells, we infected Bcap37, SGC7901 and WI-38 cells with the lentiviral vector and then treated them with increasing concentrations of $\mathrm{dFdC}$ $(0-10 \mu \mathrm{M})$ for 5 days. Subsequently, the cell viability was determined using the MTT cytotoxicity assay. Cell viability was decreased in cancer cells (Bcap37 and SGC7901) in the presence of $\mathrm{dFdC}$, demonstrating the cytotoxicity of the Lenti-hTERT-dNK/dFdC system (Fig. 4A). In contrast, slight cytotoxicity was observed in the WI-38 cells in the presence of dFdC. As the control, we observed significant cytotoxicity in the cells infected with Lenti-CMV-dNK/dFdC. The cell survival rate of Bcap37 and SGC7901 cells was $~ 80 \%$ with $0.001 \mu \mathrm{M}$ of $\mathrm{dFdC}, \sim 60 \%$ with 0.01 and $0.1 \mu \mathrm{M}$ of $\mathrm{dFdC}$, and $\sim 40 \%$ with $10 \mu \mathrm{M}$ of $\mathrm{dFdC}$. However, data showed that the normal fibroblast cells (WI-38) infected with the LentihTERT-dNK/dFdC system were resistant to dFdC. Its survival rate remained the same as before ( $\sim 90 \%$ of cells survived even with $10 \mu \mathrm{M}$ of $\mathrm{dFdC}$ ). These results illustrated that cancer cells responded to $\mathrm{dFdC}$ in a dose-dependent manner from 0.001 to $10 \mu \mathrm{M}$ in the Lenti-hTERT-dNK/dFdC system, whereas the normal fibroblast cells were completely unaffected within that 

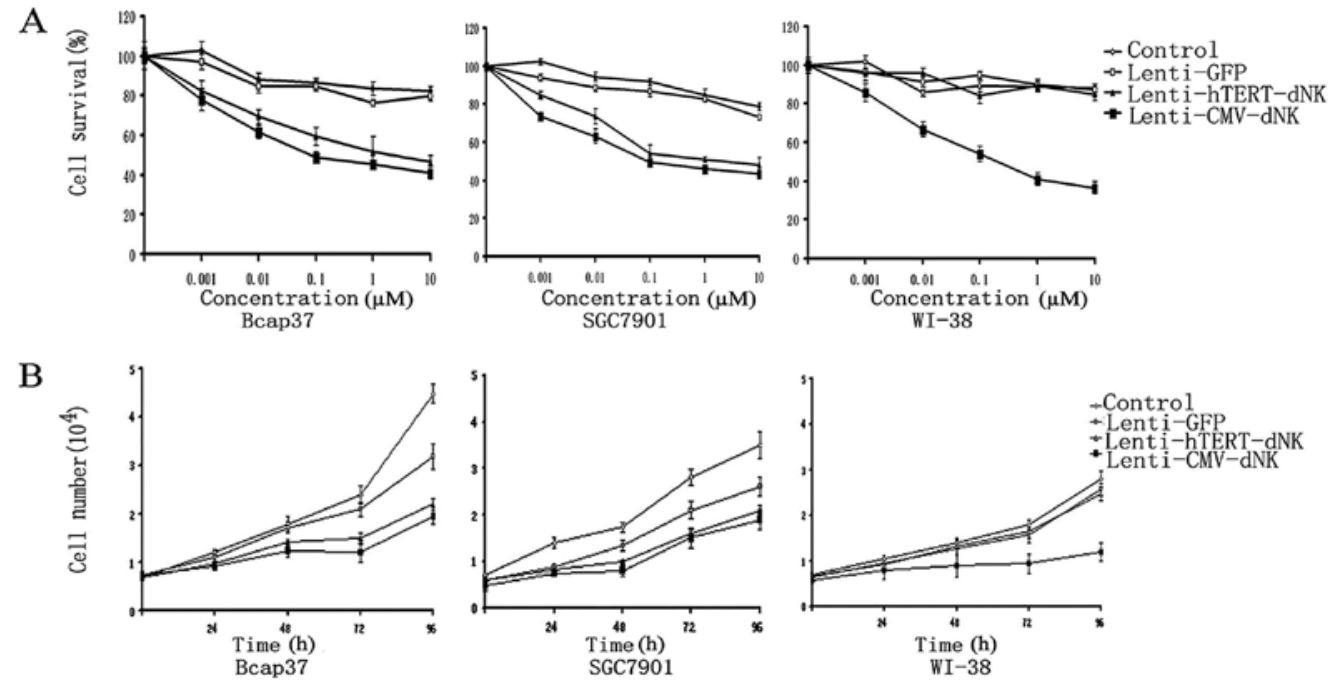

Figure 4. Cell cytotoxicity and proliferation of $D m-d N K$ with dFdC. (A) MTT analysis of cell viability was performed to determine the selective cell killing of $D m-d N K$ with lentivirus (Lenti-GFP, Lenti-hTERT-dNK and Lenti-CMV-dNK) combined with dFdC treatment at various doses. Bcap37 and SGC7901 cells infected with Lenti-hTERT-dNK had similar sensitivity to dFdC, whereas WI-38 cells were nearly unaffected by dFdC under the same conditions. Each experiment was conducted in triplicate. (B) Proliferation tests were conducted to determine the cell number from 3 cell lines after infection with lentivirus. The cells were incubated for 4 days with $\mathrm{dFdC}$, and the average cell number was obtained from three experiments.

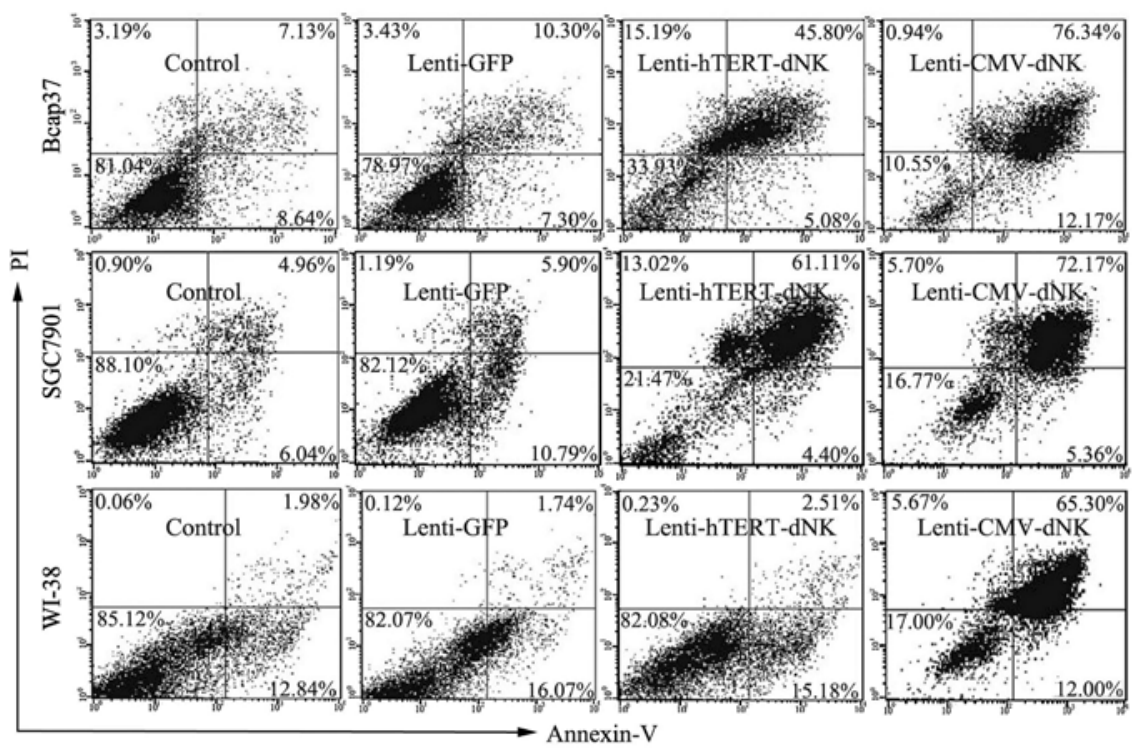

Figure 5. Flow cytometric analysis of Dm- $d N K$ expression in cancer cell lines (Bcap37 and SGC7901) and a normal cell line (WI-38) when infected with lentivirus vector and stimulated with $1 \mu \mathrm{M} \mathrm{dFdC}$. Bcap37 and SGC7901 cells infected with Lenti-hTERT-dNK both showed induction of toxicity through the greatest selective apoptosis in cancer cells.

dose range. These data suggest that the Lenti-hTERT-dNK/ $\mathrm{dFdC}$ system is able to selectively kill hTERT-positive cells in vitro.

Compared with $\mathrm{dFdC}$-induced cytotoxicity in cancer cells infected with Lenti-hTERT-dNK and Lenti-CMV-dNK, the cytotoxicity of the Lenti-hTERT-dNK/dFdC system was weaker than that of the Lenti-CMV-dNK/dFdC system. This also occurred at low concentration of $\operatorname{dFdC}(0.01 \mu \mathrm{M}, \mathrm{P}<0.05)$ in both Bcap37 and SGC7901 cells. Moreover, it was noteworthy that the cytotoxicity of the Lenti-hTERT-dNK/dFdC system was not as weaker as we expected. For example, when the $\mathrm{dFdC}$ concentration was $1 \mu \mathrm{M}$, the difference was not statistically significant $(\mathrm{P}>0.05)$ between the two cancer cell lines.

Conversely, the cell proliferation was apparently inhibited after the treatment with $1 \mu \mathrm{M}$ of $\mathrm{dFdC}$ in the cancer cells infected with Lenti-hTERT-dNK or Lenti-CMV-dNK (Fig. 4B). During the proliferation of WI-38 cells, we did not observe significant inhibition of cell growth in the LentihTERT-dNK/dFdC system (up to 4 days).

In our present study, we performed quantitative flow cytometry in order to determine the percentage of apoptotic cells. Cells infected with the Lenti-CMV-dNK/dFdC system showed the highest percentage of apoptosis, ranging from 77.3 


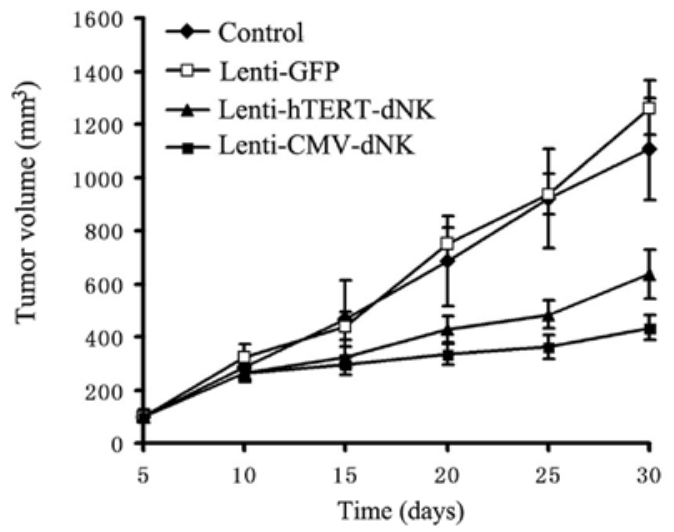

Figure 6. Lenti-hTERT-dNK/dFdC inhibited tumor growth in vivo. Bcap37 xenograft tumors in nude mice were treated by PBS, Lenti-GFP, LentihTERT-dNK or Lenti-CMV-dNK plus dFdC, respectively. Tumor size was monitored every 5 days using calipers, and tumor volume is shown. Data are presented as means $\pm \operatorname{SD}(n=6)$.

(WI-38) to $88.51 \%$ (Bcap37). In the Lenti-hTERT-dNK/dFdC system, Bcap37 and SGC7901 cells both showed a higher percentage of apoptosis (50.88 and $65.11 \%$, respectively) (Fig. 5). Conversely, the percentage of apoptosis was only $17.69 \%$ in the human embryonic lung fibroblast WI-38 cells, indicating that $D m-d N K$ had minimum contribution to the cytotoxic effects on normal cells. In addition, the WI-38 cells infected with the Lenti-hTERT-dNK/dFdC system showed nearly an identical apoptosis ratio compared with the control cells not expressing $D m-d N K$ (17.69 and $14.82 \%$, respectively). These findings were consistent with the previous observation (Fig. 4). Taken together, these data demonstrated that the LentihTERT-dNK/dFdC system efficiently induced the apoptosis of cancer cells.

Antitumor efficacy of Lenti-hTERT-dNK/dFdC in vivo. Since our in vitro data clearly indicated that Lenti-hTERT-dNK/ $\mathrm{dFdC}$ had specific cytopathic effects on tumor cells, we further examined the therapeutic potential on nude mice in order to identify whether the Lenti-hTERT-dNK/dFdC system was able to specifically inhibit tumor growth in vivo.

Either Lenti-hTERT-dNK or Lenti-CMV-dNK combined with $\mathrm{dFdC}$ treatment resulted in a significant inhibition of tumor growth in vivo compared with PBS or Lenti-GFP combined with $\mathrm{dFdC}$ treatment (Fig. 6). At Day 30, the mean volume of tumors obtained by PBS or Lenti-GFP injection combined with $\mathrm{dFdC}$ $\left(1,107.64 \pm 193.10\right.$ and $1,262.25 \pm 100.84 \mathrm{~mm}^{3}$, respectively) was larger than that of Lenti-hTERT-dNK combined with dFdC $\left(636.96 \pm 92.39 \mathrm{~mm}^{3}\right)(\mathrm{P}<0.05)$. Moreover, Lenti-hTERT-dNK combined with $\mathrm{dFdC}$ did not show an improved therapeutic benefit in terms of tumor volume on Day 30 compared with Lenti-CMV-dNK combined with $\mathrm{dFdC}\left(435.54 \pm 46.58 \mathrm{~mm}^{3}\right)$ $(\mathrm{P}<0.05)$.

\section{Discussion}

Gene delivery with a viral vector is a promising approach for the treatment of human cancer. Studies on suicide gene therapy combining herpes simplex virus thymidine kinase gene transfer and ganciclovir treatment have been widely investigated in human breast, gastric, colon, prostate and bladder cancer cells and hepatocarcinoma cells (11-16). The feasibility of suicide gene strategy has been demonstrated. However, subsequent studies have also shown that the use of the gene strategy is limited due to its poor delivery efficiency and targeted delivery problems. Therefore, an improved therapeutic strategy is required.

In our present study, we focused on the enzyme $D m-d N K$. In contrast to HSV-1 TK and other enzymes, it has a broad substrate specificity and a high catalytic rate when transferred in human cells. A previous study has shown that retroviral transduction with encoding $D m-d N K$ has cytotoxicity to several nucleoside analogs when expressed in human cells (17). However, low efficiency of the transgene and some vectorinduced toxicity are still obstacles. Therefore, it is essential to design the long-term gene expression with stable integration of the transgene. Recently, lentiviral vectors have generated novel perspectives for gene therapy, and they have become a tool for gene transfer in a broad field (18). Due to their high delivery efficiency and long-term expression of the transgene, lentiviral vectors appear to be promising candidates and can be used in clinics to cure acquired disorders. To the best of our knowledge, our study was the first to express $D m-d N K$ using a lentiviral vector. It has been reported that $D m-d N K$ expression together with $\mathrm{dFdC}$ treatment exerts a negative effect on cell survival via adenovirus. However, its effect via lentivirus remains unclear. Therefore, we developed a lentiviral vector expressing $D m-d N K$ and further investigated its suitability for medical therapy.

Another important consideration is the target of the suicide gene. It is widely accepted that hTERT is expressed in a vast majority of tumors, and its expression levels are associated with the malignancy of different types of cancer cells. The absence of hTERT results in tumor cell senescence and hence shortens the life-span of cells. In order to obtain a tumor-specific gene delivery system, we developed a lentiviral vector expressing $D m-d N K$ under the control of the hTERT promoter instead of the CMV promoter, whereas a CMV promoter cassette was designed as a control. We performed functional studies aimed at targeted killing of cancer cells by $D m-d N K$ transfer, and our data confirmed that the constitutive version can be used to induce apoptosis in cancer cells. Our study successfully demonstrated that $D m-d N K$ expression at the mRNA and protein levels under the control of the hTERT promoter was apparent in cancer cells, whereas its expression was low or absent in the normal cells. As far as the gene expression regulated by the CMV promoter was concerned, the expression of $D m-d N K$ was obvious in all cells.

It is widely accepted that cancer is promoted by an accumulation of multiple genetic and epigenetic alterations. In cancer gene therapy, it has been recognized as a crucial step to target the expression of suicide genes in a wide range of human malignant cancer cells while avoiding severe side effects in normal cells. The activity of the hTERT promoter is significantly higher in most telomerase-positive cells than that in telomerase-negative cells $(19,20)$. It is feasible to drive the expression of many therapeutic genes by the hTERT promoter, such as Bax, caspase-8 and FADD (21). In our present study, the hTERT promoter also showed the potential for targeted killing of cancer cells. However, the activity of the hTERT 
promoter in most cancer cells is suboptimal and usually weaker than that of commonly used promoters, such as the CMV promoter, SV40 promoter and RSV-LTR. Therefore, the mechanisms by which the promoter performs so differentially among cells remains unclear. Further investigation is required to clarify the mechanisms before this strategy is used as a routine clinical practice.

Previous reports have indicated that a limited number of key single amino acid residues highlight the importance of substrate specificity (22). In 2011, Zhu et al (23) discovered that the catalytic rates are relatively increased by the alteration at the sites of $244 \mathrm{E}, 245 \mathrm{~S}, 251 \mathrm{~S}$ and $252 \mathrm{R}$ of the last 10 amino acids. Based on these studies, we further investigated its important structural features and structure-function relationships.

Recently, a wide variety of other gene therapies have been investigated in many malignancies, such as antiangiogenic, tumor-suppressor, cytokine-based and oxidative-based gene therapy (24). In addition, cell cycles regulate growth and differentiation. The regulation of the different cell cycles is another hallmark of cancer control (25). Suicide gene therapies can be combined with these gene therapies, and the combination strategy may provide new potentials to deal with malignancies in future clinical application.

In summary, our study demonstrated that the recombinant suicide gene lentiviral $D m-d N K$ driven by cancer-associated hTERT promoter combined with $\mathrm{dFdC}$ for cancer gene therapy was an effective and promising treatment to use. It is conceivable that wide clinical applications may eventually be achieved through the development of this suicide gene strategy.

\section{Acknowledgements}

This study was supported by grants from the National Natural Science Foundation of China (nos. 81071900 and 81172199), the Scientific Research Foundation for Returned Scholars, the Ministry of Education of China (2008) and the Hi-Tech Research Development Program of China (863 program, 2006AA02Z493).

\section{References}

1. Bertoli A, Franco M, Balzarini J, Johansson M and Karlsson A: Altered deoxyribonucleotide pools in T-lymphoblastoid cells expressing the multisubstrate nucleoside kinase of Drosophila melanogaster. FEBS J 272: 3918-3928, 2005.

2. Knecht W, Mikkelsen NE, Clausen AR, et al: Drosophila melanogaster deoxyribonucleoside kinase activates gemcitabine. Biochem Biophys Res Commun 382: 430-433, 2009.

3. Ma S, Zhao L, Zhu Z, et al: The multisubstrate deoxyribonucleoside kinase of Drosophila melanogaster as a therapeutic suicide gene of breast cancer cells. J Gene Med 13: 305-311, 2011.

4. Zhu Z, Mao L, Zhao L, et al: Synergistic therapeutic effect in gastric cancer cells produced by oncolytic adenovirus encoding Drosophila melanogaster deoxyribonucleoside kinase. Cancer Biol Ther 11: 874-882, 2011.

5. Ito M, Suda Y, Harashima $\mathrm{H}$ and Kamiya $\mathrm{H}$ : Cytotoxic effect of Drosophila deoxynucleoside kinase gene on replicating plasmid in HeLa cells. Biol Pharm Bull 33: 1223-1227, 2010.
6. Nishino K, Osaki T, Kumagai T, et al: Adenovirus-mediated gene therapy specific for small cell lung cancer cells using a Myc-Max binding motif. Int J Cancer 91: 851-856, 2001.

7. Yu ST, Li C, Lu MH, et al: Noninvasive and real-time monitoring of the therapeutic response of tumors in vivo with an optimized hTERT promoter. Cancer 118: 1884-1893, 2012.

8. Yu B, Zhang Y, Zhan Y, et al: Co-expression of herpes simplex virus thymidine kinase and Escherichia coli nitroreductase by an hTERT-driven adenovirus vector in breast cancer cells results in additive antitumor effects. Oncol Rep 26: 255-264, 2011.

9. Ma S, Qu W, Mao L, et al: Antitumor effects of oncolytic adenovirus armed with Drosophila melanogaster deoxyribonucleoside kinase in colorectal cancer. Oncol Rep 27: 1443-1450, 2012.

10. Sandrini MP, Clausen AR, On SL, Aarestrup FM, MunchPetersen B and Piskur J: Nucleoside analogues are activated by bacterial deoxyribonucleoside kinases in a species-specific manner. J Antimicrob Chemother 60: 510-520, 2007.

11. Anderson LM, Swaminathan S, Zackon I, Tajuddin AK, Thimmapaya B and Weitzman SA: Adenovirus-mediated tissuetargeted expression of the HSVtk gene for the treatment of breast cancer. Gene Ther 6: 854-864, 1999.

12. Tang Q, Zhang D, Wan M and Jin L: Experimental study of the RV-HSV-TK/GCV suicide gene therapy system in gastric cancer. Cancer Biother Radiopharm 22: 755-761, 2007.

13. Pan JG, Zhou X, Luo R and Han RF: The adeno-associated virusmediated HSV-TK/GCV suicide system: a potential strategy for the treatment of bladder carcinoma. Med Oncol: Oct 20, 2011 (Epub ahead of print).

14. Zheng FQ, Xu Y, Yang RJ, et al: Combination effect of oncolytic adenovirus therapy and herpes simplex virus thymidine kinase/ ganciclovir in hepatic carcinoma animal models. Acta Pharmacol Sin 30: 617-627, 2009.

15. Ahn M, Lee SJ, Li X, et al: Enhanced combined tumor-specific oncolysis and suicide gene therapy for prostate cancer using M6 promoter. Cancer Gene Ther 16: 73-82, 2009.

16. Zhang JF, Wei F, Wang HP, et al: Potent antitumor activity of telomerase-dependent and HSV-TK armed oncolytic adenovirus for non-small cell lung cancer in vitro and in vivo. J Exp Clin Cancer Res 29: 52, 2010.

17. Zheng X, Johansson M and Karlsson A: Retroviral transduction of cancer cell lines with the gene encoding Drosophila melanogaster multisubstrate deoxyribonucleoside kinase. J Biol Chem 275: 39125-39129, 2000

18. Trono D: Lentiviral vectors: turning a deadly foe into a therapeutic agent. Gene Ther 7: 20-23, 2000.

19. Takakura M, Kyo S, Kanaya T, et al: Cloning of human telomerase catalytic subunit (hTERT) gene promoter and identification of proximal core promoter sequences essential for transcriptional activation in immortalized and cancer cells. Cancer Res 59: 551-557, 1999.

20. Cong YS, Wen J and Bacchetti S: The human telomerase catalytic subunit hTERT: organization of the gene and characterization of the promoter. Hum Mol Genet 8: 137-142, 1999.

21. Gu J and Fang B: Telomerase promoter-driven cancer gene therapy. Cancer Biol Ther 2 (Suppl 1): S64-S70, 2003.

22. Knecht W, Sandrini MP, Johansson K, Eklund H, MunchPetersen B and Piskur J: A few amino acid substitutions can convert deoxyribonucleoside kinase specificity from pyrimidines to purines. EMBO J 21: 1873-1880, 2002.

23. Zhu Z, Ma S, Zhao L, et al: Adenovirus-mediated Drosophila melanogaster deoxyribonucleoside kinase mutants combined with gemcitabine harbor a safe cancer treatment profile. Int J Oncol 38: 745-753, 2011.

24. Zhang C, Wang QT, Liu H, Zhang ZZ and Huang WL: Advancement and prospects of tumor gene therapy. Chin $\mathrm{J}$ Cancer 30: 182-188, 2011.

25. Abate-Daga D, Garcia-Rodriguez L, Sumoy L and Fillat C: Cell cycle control pathways act as conditioning factors for TK/GCV sensitivity in pancreatic cancer cells. Biochim Biophys Acta 1803: $1175-1185,2010$. 